\title{
Efficacy of S-1 monotherapy for non-small cell lung cancer after the failure of two or more prior chemotherapy regimens
}

\author{
OSAMU TAKAKUWA ${ }^{1}$, TETSUYA OGURI ${ }^{1}$, KEN MAENO $^{1}$, HIROAKI OZASA ${ }^{1}$, \\ YASUHITO IWASHIMA ${ }^{1}$, MIKINORI MIYAZAKI ${ }^{1}$, HIDEHARU KUNII ${ }^{2}$, YUKO TAKANO ${ }^{2}$, \\ TOSHIYUKI MORI ${ }^{2}$, SHIGEKI SATO ${ }^{1}$ and RYUZO UEDA ${ }^{1}$

\begin{abstract}
${ }^{1}$ Department of Medical Oncology and Immunology, Nagoya City University Graduate School of Medical Sciences, Nagoya 467-8601; ${ }^{2}$ Department of Respiratory Medicine, Gifu Prefectural Tajimi Hospital, Gifu 507-8522, Japan
\end{abstract}

Received June 2, 2009; Accepted August 28, 2009

DOI: 10.3892/ol_00000027

\begin{abstract}
The efficacy and safety of S-1 monotherapy for patients with advanced or recurrent non-small cell lung cancer (NSCLC) after the failure of two or more prior chemotherapy regimens were investigated. Records of 36 patients with advanced or recurrent NSCLC who received S-1 monotherapy between January 2005 and December 2008, following the failure of previous chemotherapy, were reviewed retrospectively at two institutions. S-1 was given orally twice daily on days 1-28 every six weeks; the dose was based on body surface area. The median number of prior chemotherapy regimens was three (range 2-5), and that of courses given per patient was two (range 1-10). No patient achieved complete response, 4 patients (11.1\%) achieved partial response, 10 patients (27\%) had stable disease and 18 patients $(50 \%)$ had progressive disease. The median progression-free survival was 3 months and the median overall survival was 15.2 months. No grade 4 hematological toxicity was noted. Grade $\geq 3$ non-hematological toxicities were observed in 5 patients $(13.9 \%)$. No deaths related to S-1 monotherapy occurred. S-1 monotherapy exhibits activity with acceptable toxicity as third-line or subsequent chemotherapy for advanced NSCLC.
\end{abstract}

\section{Introduction}

Lung cancer is the leading cause of cancer-related deaths worldwide and non-small cell lung cancer (NSCLC) accounts for approximately $80 \%$ of lung cancer. At presentation, only $30 \%$ of NSCLCs are resectable, while the remaining $70 \%$ of patients with NSCLC have advanced disease. Subsequently,

Correspondence to: Dr Tetsuya Oguri, Department of Medical Oncology and Immunology, Nagoya City University Graduate School of Medical Sciences, 1 Kawasumi, Mizuho-cho, Mizuho-ku, Nagoya 467-8601, Japan

E-mail: t-oguri@med.nagoya-cu.ac.jp

Key words: S-1, non-small cell lung cancer, chemotherapy failure, third-line treatment these patients are potential candidates for systemic treatment only.

Platinum-based chemotherapy is the recommended first-line treatment for advanced NSCLC. This type of chemotherapy prolongs survival, improves symptom control and results in a superior quality of life compared to best supportive care. Benefits of second-line chemotherapy have been substantiated by randomized trials using docetaxel $(1,2)$, pemetrexed (3) and erlotinib (4). However, the response rate was low, and it is well known that almost all patients with advanced NSCLC ultimately progress or relapse. Thus, more treatment is needed to control the disease and improve quality of life for patients suffering from NSCLC.

$\mathrm{S}-1$ is a novel oral fluoropyrimidine derivative consisting of tegafur (FT), which is a prodrug of 5-fluorouracil (5-FU) and the modulators 5-chloro-2,4-dihydroxypyridine (CDHP) and potassium oxonate (Oxo), at a molar ratio of 1:0.4:1 (5). CDHP is a reversible competitive inhibitor of dihydropyrimidine dehydrogenase, an enzyme involved in the degradation of 5-FU. The degradation of FT-derived 5-FU is inhibited by CDHP, resulting in enhancement of the antitumor effect $(6,7)$. Oxo reduces the gastrointestinal toxicity of 5-FU. After its oral administration, Oxo is distributed selectively to the small and large intestines. In the intestines, Oxo inhibits the phosphorylation of 5-FU to fluoropyrimidine monophosphate which is catalyzed by orotate phosphoribosyltransferase within the gastrointestinal mucosal cells, thereby reducing the incidence of diarrhea (8).

S-1 is reportedly effective in the treatment of certain types of solid cancer, such as gastric (9), breast (10), colorectal (11) and pancreatic (12). As regards the efficacy of S-1 monotherapy for NSCLC, phase II trials showed an overall response rate of $22 \%$ and a median survival time (MST) of 10.2 months (13). Furthermore, Furuse et al reported that there was a low frequency of irreversible, severe or unexpected toxicities (14). S-1 monotherapy may be a treatment regimen suitable for NSCLC patients after the failure of two or more prior chemotherapy regimens. However, at present, there is no defined role for S-1 chemotherapy after second-line chemotherapy for NSCLC patients.

To investigate the efficacy of S-1 monotherapy, records of patients with advanced or recurrent NSCLC who received S-1 
following a previously failed response to chemotherapy were retrospectively analyzed.

\section{Patients and methods}

Patient selection. The study included patients with advanced or recurrent NSCLC who received S-1 monotherapy following the failure of previous chemotherapy at Nagoya City University Hospital and Gifu Prefectural Tajimi Hospital between January 2005 and December 2008. Patients who fulfilled the following selection criteria were included: failure of two or more chemotherapy regimens, Eastern Cooperative Oncology Group (ECOG) performance status (PS) of 2 or better, no other active malignancies and adequate bone marrow, renal and hepatic function. These patients were observed until March 15, 2009.

Treatment method. S-1 was administered orally, twice daily, after meals, on days 1-28 every six weeks. The dose of S-1 was determined to be $80 \mathrm{mg} /$ day (body surface area $<1.25 \mathrm{~m}^{2}$ ), $100 \mathrm{mg} /$ day (body surface area $\geq 1.25$ and $<1.50 \mathrm{~m}^{2}$ ) or $120 \mathrm{mg} /$ day (body surface area $\geq 1.50 \mathrm{~m}^{2}$ ). The schedule and dose for each patient were modified by the physician according to the medical condition of the patient, as well as toxicity observed in the previous chemotherapy regimen or courses.

Evaluation and statistical analysis. Patient medical records were retrospectively analyzed. Responses were assessed with the use of the Response Evaluation Criteria in Solid Tumors (15). Progression-free (PFS) and overall survival were measured from the start of S-1 therapy until progressive disease and death, respectively. Survival results were analyzed using the Kaplan-Meier method. Toxicity was assessed according to the common toxicity criteria of the National Cancer Institute version 2.0.

\section{Results}

Patient numbers and characteristics. Table I shows patient characteristics. The median age was 68 years (range 41-76). There were 27 male $(75 \%)$ and 9 female $(25 \%)$ patients of whom the majority (33 patients, 91.6\%) had an ECOG PS of 1 or 0. Nineteen patients $(52.7 \%)$ had adenocarcinoma and 14 patients (38.9\%) had squamous cell carcinoma. Six patients $(16.7 \%)$ had clinical stage IIIB and $28(77.8 \%)$ had stage IV disease. Two regimens were given to 10 patients $(27.8 \%), 3$ regimens were given to 13 patients $(36.1 \%)$ and $\geq 4$ to 13 patients $(36.1 \%)$. Of a total of 36 patients, $31(86.1 \%)$ had previously received platinum-based chemotherapy and 27 patients (75\%) had previously received both platinum-based chemotherapy and docetaxel. Three female patients with adenocarcinoma had already received epidermal growth factor receptor tyrosine kinase inhibitor (gefitinib or erlotinib). Twenty-one patients (58.3\%) received subsequent chemotherapy following the termination of S-1 monotherapy.

Treatment delivery. In total, 102 courses were given ( 2 median courses per patient; range 1-10). The 27 male patients (75\%) started S-1 monotherapy at the established treatment dose, while the 9 female patients $(25 \%)$ were started at a reduced dose. During S-1 monotherapy, 5 patients (13.9\%) required a
Table I. Characteristics of the 36 treated patients.

\begin{tabular}{|c|c|}
\hline Characteristic & No. of patients $(\%)$ \\
\hline Median age, years (range) & $68(41-76)$ \\
\hline \multicolumn{2}{|l|}{ Gender } \\
\hline Male & $27(75.0)$ \\
\hline Female & $9(25.0)$ \\
\hline \multicolumn{2}{|l|}{ Performance status (ECOG) } \\
\hline 0 & $12(33.3)$ \\
\hline 1 & $21(58.3)$ \\
\hline 2 & $3(8.3)$ \\
\hline \multicolumn{2}{|l|}{ Smoking history } \\
\hline Current smoker or ever smoker & $22(61.1)$ \\
\hline Never smoked & $11(30.6)$ \\
\hline Unknown & $3(8.3)$ \\
\hline \multicolumn{2}{|l|}{ Histology } \\
\hline Adenocarcinoma & $19(52.8)$ \\
\hline Squamous cell carcinoma & $14(38.9)$ \\
\hline NSCLC not specified & $3(8.3)$ \\
\hline \multicolumn{2}{|l|}{ Stage of disease } \\
\hline IIIA & $2(5.6)$ \\
\hline IIIB & $6(16.7)$ \\
\hline IV & $28(77.8)$ \\
\hline \multicolumn{2}{|l|}{ Number of prior regimens } \\
\hline 2 & $10(27.8)$ \\
\hline 3 & $13(36.1)$ \\
\hline 4 & $10(27.8)$ \\
\hline 5 & $3(8.3)$ \\
\hline
\end{tabular}

NSCLC, non-small cell lung cancer; ECOG, Eastern Cooperative Oncology Group.

change in the treatment method: the period of S-1 administration was shortened in 4 patients, while the $S-1$ dose was reduced in 1 patient. In 3 patients, S-1 monotherapy was still continued at the end of the observation period. Thirty-three patients stopped S-1 treatment due to disease progression (27 patients), adverse events (5 patients) and refusal of treatment (1 patient).

Toxicity. The adverse events observed in the 36 patients are shown in Table II. Grade 3 anemia was observed in 1 patient, while 2 patients had grade 3 neutropenia. Grade 4 hematological toxicities did not occur. Non-hematological toxicities of grade $\geq 3$ were observed in 5 patients (13.9\%): elevation of the serum amylase level in 2 patients, interstitial pneumonia in 1 patient and pneumonitis caused by infection in 2 patients. Interstitial pneumonia of any grade was observed in 3 patients, two of whom were diagnosed as having acute exacerbation of idiopathic pulmonary fibrosis (IPF) by their physician. No deaths related to S-1 monotherapy occurred. 
Table II. Toxicities for the 36 treated patients.

\begin{tabular}{|c|c|c|c|c|c|}
\hline \multirow[t]{2}{*}{ Toxicity } & \multicolumn{4}{|c|}{ Grade } & \multirow[b]{2}{*}{$\geq 3(\%)$} \\
\hline & 1 & 2 & 3 & 4 & \\
\hline Leukopenia & 1 & 5 & 1 & 0 & 2.8 \\
\hline Neutropenia & 1 & 1 & 2 & 0 & 5.6 \\
\hline Anemia & 3 & 10 & 1 & 0 & 2.8 \\
\hline Thrombocytopenia & 12 & 3 & 0 & 0 & 0 \\
\hline Anorexia & 9 & 5 & 0 & 0 & 0 \\
\hline Fatigue & 6 & 1 & 0 & 0 & 0 \\
\hline Diarrhea & 3 & 0 & 0 & 0 & 0 \\
\hline Nausea & 6 & 2 & 0 & 0 & 0 \\
\hline Vomiting & 1 & 1 & 0 & 0 & 0 \\
\hline Stomatitis & 2 & 0 & 0 & 0 & 0 \\
\hline Rash & 5 & 1 & 0 & 0 & 0 \\
\hline Hyperbilirubinemia & 4 & 0 & 0 & 0 & 0 \\
\hline $\mathrm{AST}^{\mathrm{a}}$ & 7 & 1 & 0 & 0 & 0 \\
\hline $\mathrm{ALT}^{\mathrm{a}}$ & 7 & 1 & 0 & 0 & 0 \\
\hline Creatinine $^{a}$ & 0 & 1 & 0 & 0 & 0 \\
\hline Amylase $^{\mathrm{a}}$ & 0 & 0 & 1 & 1 & 5.6 \\
\hline Interstitial pneumonitis & 1 & 1 & 0 & 1 & 2.8 \\
\hline Pneumonitis & 0 & 0 & 2 & 0 & 5.6 \\
\hline
\end{tabular}

ancreased levels of AST, aspartate aminotransferase; ALT, alanine aminotransferase; as well as creatinine or amylase.

Antitumor activity. Treatment response was not assessed in 4 patients because treatment was terminated during the first course due to toxicity or refusal. In the remaining 32 patients, no patient achieved complete response (CR), 4 patients $(11.1 \%)$ achieved partial response (PR), 10 patients (27.8\%) had stable disease (SD) and 18 patients (50\%) had progressive disease. The disease control rate (DCR), as well as the rate of patients who had achieved CR, PR or SD was $38.9 \%$. The 36 patients exhibited a median PFS time of 3 months (Fig. 1) and the overall MST was 15.2 months (Fig. 2).

\section{Discussion}

This study retrospectively analyzed the efficacy and safety of S-1 monotherapy in patients with advanced or recurrent NSCLC who received S-1 after previous chemotherapy. S-1 monotherapy thus exhibited activity with acceptable toxicities.

Concerning the outcome of patients who received two chemotherapy regimens, including platinum and docetaxel for recurrent NSCLC, Massarelli et al reported that response rates decreased with each line of treatment (third-line, $2.3 \%$; fourth line, 0\%). DCR also decreased (third-line, 30.2\%; fourth-line, 21.4\%), while MST from the last treatment was 4 months (16). Shepherd et al reported that the overall response rate was $9.9 \%$ in advanced NSCLC patients treated with erlotinib after two or three prior regimens (4). Igawa et al reported the efficacy of amrubicin for NSCLC patients after the failure of $\geq 2$ prior chemotherapy regimens; the overall response rate was $10.2 \%$ and MST was 4.8 months (17). The results of the present study

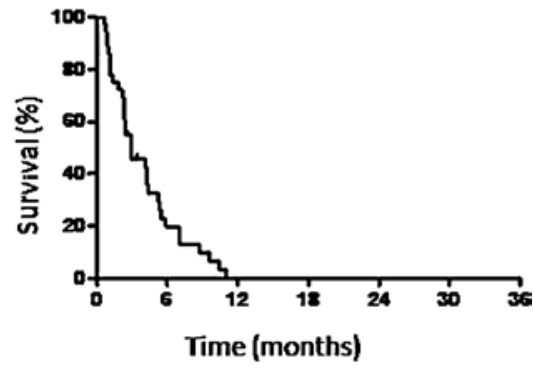

Figure 1. Kaplan-Meier curve for progression-free survival in the 36 patients treated with S-1 monotherapy.

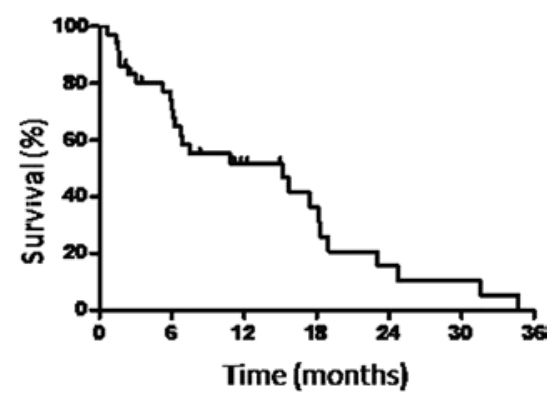

Figure 2. Kaplan-Meier curve for overall survival in the 36 patients treated with S-1 monotherapy.

indicate that S-1 monotherapy had a variable clinical outcome for patients with advanced or recurrent NSCLC who received S-1 after previous chemotherapy.

As regards the toxicities of S-1 monotherapy for NSCLC patients, previous phase II studies showed that grade 3 or 4 neutropenia was observed in 5.4-6.8\% of patients. Grade $\geq 3$ non-hematological toxicities, such as anorexia, fatigue or diarrhea were observed in approximately $5 \%$ of patients $(13,14)$. In the current study, grade 3 leukopenia and anemia were observed in only 1 patient $(2.8 \%)$ each. Grade 3 neutropenia was observed in 2 patients $(5.6 \%)$. Grade 4 hematological toxicity was not observed. Grade $\geq 3$ non-hematological toxicities were observed in 5 patients $(13.9 \%)$. Given that patients in the present study received S-1 as third-line or later chemotherapy, the toxicities observed were sufficiently acceptable.

Interstitial pneumonia was observed in 3 patients $(8.3 \%)$, two of whom were diagnosed as having an acute exacerbation of IPF by their physician. Interstitial pneumonia-related S-1 toxicity was reported to be relatively rare (18). It is unclear whether the interstitial pneumonia noted in patients was a direct result of S-1 treatment, but careful consideration is needed when $S-1$ is given to patients with IPF.

It is important to avoid severe toxicity and make an effort to obtain a reasonable quality of life for advanced NSCLC patients previously treated with chemotherapy. Oral chemotherapy is beneficial in terms of patient quality of life and cost-effectiveness (19). In the present study, 15 patients $(41.7 \%)$ received S-1 chemotherapy at a reduced dose or with a shortened length of administration because of toxicity. A prospective study aimed at elucidating the efficacy of this agent, and investigating the appropriate treatment setting for NSCLC following the failure of prior chemotherapy regimens is warranted. 


\section{References}

1. Shepherd FA, Dancey J, Ramlau R, et al: Prospective randomized trial of docetaxel versus best supportive care in patients with nonsmall-cell lung cancer previously treated with platinum-based chemotherapy. J Clin Oncol 18: 2095-2103, 2000.

2. Fossella FV, DeVore R, Kerr RN, et al: Randomized phase III trial of docetaxel versus vinorelbine or ifosfamide in patients with advanced non-small cell lung cancer previously treated with platinum-containing chemotherapy regimens. The TAX 320 Non-Small Cell Lung Cancer Study Group. J Clin Oncol 18 : 2354-2362, 2000.

3. Hanna N, Shepherd FA, Fossella FV, et al: Randomized phase III trial of pemetrexed versus docetaxel in patients with non-small cell lung cancer previously treated with chemotherapy. J Clin Oncol 22: 1589-1597, 2004.

4. Shepherd FA, Rodrigues Pereira J, Ciuleanu T, et al: Erlotinib in previously treated non-small cell lung cancer. N Engl J Med 353: 123-132, 2005

5. Shirasaka T, Nakano K, Takechi T, et al: Antitumor activity of $1 \mathrm{M}$ tegafur-0.4 M 5-chloro-2,4-dihydroxypyridine-1 M potassium oxonate (S-1) against human colon carcinoma orthotopically implanted into nude rats. Cancer Res 56: 2602-2606, 1996.

6. Tatsumi K, Fukushima M, Shirasaka T and Fujii S: Inhibitory effects of pyrimidine, barbituric acid and pyridine derivatives on 5-fluorouracil degradation in rat liver extracts. Jpn J Cancer Res 78: 748-755, 1987

7. Oguri T, Achiwa H, Bessho Y, et al: The role of thymidylate synthase and dihydropyrimidine dehydrogenase in resistance to 5-fluorouracil in human lung cancer cells. Lung Cancer 49: 345-351, 2005.

8. Shirasaka T, Shimamoto $\mathrm{Y}$ and Fukushima M: Inhibition by oxonic acid of gastrointestinal toxicity of 5-fluorouracil without loss of its anti-tumor activity in rats. Cancer Res 53: 4004-4009, 1993.

9. Koizumi W, Narahara H, Hara T, et al: S-1 plus cisplatin versus S-1 alone for first-line treatment of advanced gastric cancer (SPIRITS trial): a phase III trial. Lancet Oncol 9: 215-211, 2008.
10. Saeki T, Takashima S, Sano M, et al: A phase II study of S-1 in patients with metastatic breast cancer - a Japanese trial by the S-1 Cooperative Study Group, Breast Cancer Working Group. Breast Cancer 11: 194-202, 2004.

11. Goto A, Yamada Y, Yasui H, et al: Phase II study of combination therapy with S-1 and irinotecan in patients with advanced colorectal cancer. Ann Oncol 17: 968-973, 2006.

12. Okusaka T, Funakoshi A, Furuse J, Boku N, Yamao K, Ohkawa S and Saito H: A late phase II study of S-1 for metastatic pancreatic cancer. Cancer Chemother Phamacol 61: 615-621, 2008.

13. Kawahara M, Furuse K, Segawa Y, et al: Phase II study of S-1, a novel oral fluorouracil, in advanced non-small cell lung cancer. Br J Cancer 85: 939-943, 2001.

14. Furuse K, Kawahara M, Hasegawa K, et al: Early phase II study of S-1, a new oral fluoropyrimidine, for advanced non-small cell lung cancer. Int J Clin Oncol 6: 236-241, 2001.

15. Therasse P, Arbuck SG, Eisenhauer EA, et al: New guidelines to evaluate the response to treatment in solid tumors. European Organization for Research and Treatment of Cancer, National Cancer Institute of the United States, National Cancer Institute of Canada. J Natl Cancer Inst 92: 205-216, 2000.

16. Massarelli E, Andre F, Liu DD, et al: A retrospective analysis of the outcome of patients who have received two prior chemotherapy regimens including platinum and docetaxel for recurrent non-small cell lung cancer. Lung Cancer 39: 55-61, 2003.

17. Igawa S, Takahashi T, Nakamura Y, et al: Efficacy of amrubicin for non-small cell lung cancer after failure of two or more prior chemotherapy regimens. Anticancer Res 28: 3855-3858, 2008.

18. Kurakawa E, Kasuga I, Ishizuka S, et al: Interstitial pneumonia possibly due to a novel anticancer drug, TS-1: first case report. Jpn J Clin Oncol 31: 284-286, 2001.

19. DeMario MD and Ratain MJ: Oral chemotherapy: rational and future directions. J Clin Oncol 16: 2557-2567, 1998. 